CORRIGENDA

\title{
Attention-deficit hyperactivity disorder in adults: a systematic review and meta-analysis of genetic, pharmacogenetic and biochemical studies
}

\author{
C Bonvicini, SV Faraone and C Scassellati
}

Molecular Psychiatry (2016) 21, 1643; doi:10.1038/mp.2016.128; published online 9 August 2016

Correction to: Molecular Psychiatry 21: 872-884; doi:10.1038/mp. 2016.74; Published online 24 May 2016

Updated online 09/08/16: This article was originally published under NPG's License to Publish, but has now been made available under a CCL ND 4.0 license. The PDF and HTML versions of the paper have been modified accordingly.

\begin{abstract}
(c) (i) $\odot$ This work is licensed under a Creative Commons AttributionNonCommercial-NoDerivs 4.0 International License. The images or other third party material in this article are included in the article's Creative Commons license, unless indicated otherwise in the credit line; if the material is not included under the Creative Commons license, users will need to obtain permission from the license holder to reproduce the material. To view a copy of this license, visit http:// creativecommons.org/licenses/by-nc-nd/4.0/
\end{abstract}

C The Author(s) 2016

\section{Genome-wide analysis of over 106000 individuals identifies 9 neuroticism-associated loci}

DJ Smith, V Escott-Price, G Davies, MES Bailey, L Colodro-Conde, J Ward, A Vedernikov, R Marioni, B Cullen, D Lyall, SP Hagenaars, DCM Liewald, M Luciano, CR Gale, SJ Ritchie, C Hayward, B Nicholl, B Bulik-Sullivan, M Adams, B Couvy-Duchesne, N Graham, D Mackay, J Evans, BH Smith, DJ Porteous, SE Medland, NG Martin, P Holmans, AM Mclntosh, JP Pell, IJ Deary and MC O'Donovan

Molecular Psychiatry (2016) 21, 1643-1644; doi:10.1038/mp.2016.177; published online 13 September 2016

Table 2A. Genome-wide significant index SNPs. Combined meta-analysis of UK Biobank, GS:SFHS and QIMR data sets

\begin{tabular}{|c|c|c|c|c|c|c|c|c|c|c|}
\hline Index SNP & Chr & Position & $A 1 / A 2$ & Freq & BETA (SE) & $P$ & $\begin{array}{c}\text { Direction } \\
\text { (UKBB-GS- } \\
\text { QMIR) }\end{array}$ & $\begin{array}{c}\text { Heter } \\
\text { P }\end{array}$ & Associated region & Genes \\
\hline rs490647 & 1 & 37242743 & $A / G$ & 0.227 & $0.091(0.017)$ & $5.0 \times 10^{-8}$ & +++ & 0.720 & $37219429-37261085$ & GRIK3 \\
\hline rs4653663 & 1 & 225927218 & $\mathrm{~A} / \mathrm{T}$ & 0.255 & $0.091(0.016)$ & $1.8 \times 10^{-8}$ & +++ & 0.095 & $225899639-225947638$ & ENAH, SRPQ \\
\hline rs12637928 & 3 & 110184749 & $\mathrm{~A} / \mathrm{T}$ & 0.490 & $-0.077(0.014)$ & $4.3 \times 10^{-8}$ & --- & 0.695 & $110103126-110299632$ & PVRL3 (579KB distal) \\
\hline rs62353264 & 4 & 166085805 & $\mathrm{~A} / \mathrm{T}$ & 0.986 & $-0.330(0.061)$ & $5.5 \times 10^{-8}$ & --+ & 0.158 & $166063134-166198156$ & TMEM192, KLHL2, MSMO1 \\
\hline rs12682352 & 8 & 8646246 & $\mathrm{~T} / \mathrm{C}$ & 0.525 & $0.115(0.014)$ & $9.0 \times 10^{-15}$ & +++ & 0.433 & $8301794-10831868$ & More than 10 genes \\
\hline rs12378446 & 9 & 11369213 & $\mathrm{~T} / \mathrm{C}$ & 0.791 & $0.099(0.017)$ & $9.4 \times 10^{-9}$ & +++ & 0.831 & $11131371-11880898$ & PTRD (650KB distal) \\
\hline rs4977844 & 9 & 23295899 & $\mathrm{C} / \mathrm{G}$ & 0.358 & $0.083(0.015)$ & $1.4 \times 10^{-8}$ & +++ & 0.318 & $23291526-23340616$ & ELAVL2 \\
\hline rs111433752 & 17 & 43857989 & $\mathrm{~T} / \mathrm{G}$ & 0.790 & $-0.121(0.018)$ & $6.7 \times 10^{-12}$ & --- & 0.053 & $43463493-44865603$ & More than 10 genes \\
\hline rs1187264 & 18 & 35289647 & $C / G$ & 0.136 & $0.118(0.021)$ & $9.5 \times 10^{-9}$ & +++ & 0.515 & $35287090-35413260$ & CELF4 \\
\hline
\end{tabular}

Abbreviations: Chr, chromosome; Freq, frequency; GS:SFHS, Generation Scotland: Scottish Family Health Study; Heter, heterogeneity; QIMR, Queensland Institute of Medical Research (QIMR) Berghofer Medical Research Institute; SNP, single-nucleotide polymorphism. Shown are linkage disequilibrium (LD)independent genome-wide significant SNP associations for neuroticism (sorted by genomic position according to UCSC hg19/NCBI Build 37). Column A1/A2 has the SNP alleles, with the first allele (A1) the reference allele for the frequency and $\beta$ columns. Frequency of allele 1 is calculated in the UK Biobank data set. $\mathrm{Chr}$ and Position denote the location of the index SNP. $\beta$ is linear regression coefficient for allele1, and s.e. is the standard error for $\beta$. Associated region indicates range positions of SNPs with $r^{2}>0.6$ with the index and any other genome-wide association study (GWAS) significant SNP at the locus. The final column indicates protein-coding reference sequence genes at the associated loci (see region plots in Supplementary Information) or where there are no genes at the associated locus, the nearest gene if $<1 \mathrm{Mb}$ from the locus. 